\title{
SALUD
}

\section{Caracterización de mortalidad por tumores malignos más frecuente a nivel nacional. Paraguay. Año 2006 - 2015}

\author{
Agustina Rojas Silva1', Estela Meza de Quiñonez ${ }^{1}$
}

\begin{abstract}
Resumen
Introducción: El cáncer es una de las enfermedades crónicas no transmisibles de mayor impacto por su morbilidad y mortalidad, es la segunda causa de muerte en el mundo. En el año 2015 a nivel mundial se registró 8,8 millones de defunciones, cerca del $70 \%$ de las muertes ocurrió en países de ingresos medios y bajos; en el año 2012 en las Américas se registró 1, 3 millones respectivamente. En Paraguay en el 2015 ocasiono 4 mil defunciones por esta causa, con una tasa de mortalidad de 65.9 por 100.000 habitantes, siendo la primera causa de muertes prematuras en personas con edades entre 30 y 70 años La detección, el cribado, tratamiento y cuidados paliativos son componentes fundamentales de la respuesta a las Enfermedades No Transmisibles.
\end{abstract}

Objetivos: Describir los casos de mortalidad por tumores malignos registrados en Paraguay en el periodo 2006 al 2015.

Material y Método: Se realizó un estudio descriptivo observacional con datos de estadísticas vitales que fueron remitidos por los servicios de salud a la Dirección General de Información Estratégica en Salud en el periodo 2006 al 2015. Se analizaron variables como el sexo, edad y localización anatómica. Para el cálculo de tasa se consideró el número de defunciones por tumores malignos/población por 100.000 habitantes. Para el análisis de los datos se utilizó Microsoft Excel 2013.

Resultados: En el periodo de estudio se registró 34.729 defunciones por tumores malignos, con un promedio de 3.473 casos anuales (3.270-4.072); el $52,1 \%(18.102 / 34.729)$ corresponde al sexo masculino. La tasa de mortalidad promedio por tumores en el periodo de estudio fue 55 por 100.000 habitantes. Las cuatro principales localizaciones anatómica de tumores en el sexo masculino fueron: 36,4\% (3.529/9.686) pulmón, 31,3\% (3.033/9.686) próstata, $18,5 \%(1.793)$ y tejido linfático; $13,7 \%$ (1.131/9.686) colo rectal, en el sexo femenino localización más frecuente fue: 33,0\% (3.055/9.254) mama, 27\% (2.497/9.254) cuello de uterino y $14,5 \%$ (1.344/9.254) colo rectal. En el sexo

\footnotetext{
1 Ministerio de Salud Pública y Bienestar Social, Dirección General de Vigilancia de la Salud.

Este trabajo fue presentado en Conferencia Científica Anual de AMET (Asunción Paraguay 22 al 24 de octubre2017.

E-mail: arojassilva@hotmail.com

DOI: $10.26885 /$ rcei.foro.2018.134
} 
masculino el promedio anual de fallecidos es 656 y presentaron localización a nivel de pulmón y próstata; del sexo femenino 553 fallecidas por tumores con localización en mama y cuello uterino. La mayor proporción de tumores se registran en mayores de 60 años de edad.

Conclusiones: Los casos de tumores malignos han ido en aumento presentando una casuística de fallecimiento de una mujer por día a consecuencia del cáncer de mama y cuello uterino; ambos prevenibles; en el sexo masculino se registran un promedio de dos fallecimientos por día con localización en pulmón y próstata. Se recomienda fortalecer el acceso al diagnóstico temprano, tratamiento adecuado de los casos e impulsar campaña de prevención en todos los niveles de atención, además ampliar el estudio desagregando los casos de defunciones por procedencia.

Palabras clave: tumores malignos, mortalidad, Paraguay.

\section{Referencias}

Ministerio de Salud Pública y Bienestar Social. (2016). Indicadores Básicos de Salud. Recuperado de http://portal.mspbs.gov.py/digies/wp-content/ uploads/2012/01/IBS-Paraguay-2016.pdf

Organización Mundial de la Salud. Cáncer. (2018). Recuperado de https://www. who.int/es/news-room/fact-sheets/detail/cancer

Organización Mundial de la Salud. (2018). Informe de la Comisión Mundial Independiente de Alto Nivel de la OMS para las Enfermedades No Transmisibles (ENT). Recuperado de https://www.paho.org/uru/index. php?option=com_content\&view=article\&id=1293: nuevo-informe-dela-comision-mundial-independiente-de-alto-nivel-de-la-oms-para-lasenfermedades-no-transmisibles-ent\&Itemid=451

Organización Mundial de la Salud. (2018). Enfermedades No Transmisibles. Recuperado de https://www.who.int/es/news-room/fact-sheets/ detail/noncommunicable-diseases

Organización Mundial de la Salud. (2018). La inversión en el control de las enfermedades no transmisibles genera importantes beneficios financieros y sanitarios. Recuperado de https://www.who.int/es/newsroom/detail/16-05-2018-investing-in-noncommunicable-diseasecontrol-generates-major-financial-and-health-gains 